\title{
Scattering Centers Detection and Tracking in Refocused Spaceborne SAR Images for Infrastructure Monitoring
}

\author{
Andrei Anghel, Student Member, IEEE, Gabriel Vasile, Member, IEEE, Remus Cacoveanu, Member, IEEE, \\ Cornel Ioana, Member, IEEE, Silviu Ciochina, Senior Member, IEEE, and Jean-Philippe Ovarlez, Member, IEEE
}

\begin{abstract}
Infrastructure monitoring applications can require the tracking of slowly moving points of a certain structure. Given a certain point from a structure to be monitored, in the context of available spaceborne synthetic aperture radar (SAR) products, where the image is already focused in a slant range-azimuth grid, it is not obvious if this point is the scattering center, if it is in layover or if it is visible from the respective orbit. This paper proposes a scattering centers detection and tracking procedure based on refocusing a set of SAR images on a provided high-resolution grid of the structure. The refocusing procedure is designed for high-resolution spotlight and sliding spotlight SAR images and consists of an azimuth defocusing followed by a modified backprojection algorithm on the given set of points. The scattering centers of the refocused image are detected in the 4-D tomography framework by testing if the main response is at zero elevation in the local elevation-velocity spectral distribution obtained using the Capon estimator. The mean displacement velocity is estimated from the peak response on the zero elevation axis, whereas the displacements time series for detected single scatterers is obtained as phase difference of complex amplitudes. The algorithm is tested by simulations with an emphasis on its behavior for a low number of satellite passes and applied on real data acquired with the TerraSAR-X satellite over the Puylaurent dam. The relative dis-placements between scattering regions show very good agreement with in situ measurements.
\end{abstract}

Index Terms-Interferometry, refocusing, synthetic aperture radar (SAR), tomography, 3-D Mapping.

\section{INTRODUCTION}

$\mathbf{I}$ $\mathrm{N}$ infrastructure monitoring, it can be often necessary to track the slow displacements of certain points of a given structure (building, water dam, landslide, etc.). This can be done using current spaceborne civil sensors, such as TerraSAR-X

A. Anghel is with the Department of Telecommunications, University Politehnica of Bucharest (UPB), 060032 Bucharest, Romania, and also with the Grenoble Image sPeech Signal Automatics Laboratory (GIPSA-Lab), Grenoble Institute of Technology, 38402 Grenoble, France (e-mail: andrei.anghel@ munde.pub.ro).

G. Vasile and C. Ioana are with the GIPSA-Lab, Grenoble Institute of Technology, 38402 Grenoble, France (e-mail: gabriel.vasile@gipsa-lab.grenobleinp.fr; cornel.ioana@gipsa-lab.grenoble-ino.fr).

R. Cacoveanu and S. Ciochina are with the Department of Telecommunications, University Politehnica of Bucharest (UPB), 060032 Bucharest, Romania (e-mail: cacoveanu@munde.pub.ro; silviu@comm.pub.ro).

J.-P. Ovarlez is with The French Aerospace Lab (ONERA), 91761 Palaiseau, France (e-mail: jean-philippe.ovarlez@onera.fr). and TanDEM-X provided their short wavelength of $3.1 \mathrm{~cm}$, the short revisit time of 11 days and particularly the $1-\mathrm{m}$ resolution in spotlight mode [1], [2]. Still, given a certain infrastructure element, from one acquisition geometry only one side of the structure can be observed. Due to typical sidelook effects (layover ambiguities, multipath scattering effects or shadowing) it is not always clear from what points the main response returns, where is their scattering center positioned, which of them are visible from the respective orbit and consequently which of them can be accurately monitored. If the coordinates of a number of points from the structure are known with at least centimeter accuracy (measured with GPS or LIDAR techniques), their response (if there is any) could be determined if the raw data were focused on a 3-D grid precisely containing these points. However, in most cases in the delivered products, the synthetic aperture radar (SAR) images are already focused on a slant range-azimuth grid that is not related to any specific scatterer.

Since the availability and processing of spaceborne raw synthetic aperture radar (SAR) data is not very convenient, this paper proposes a scattering centers monitoring method based on refocusing [3], [4] the SAR data on a given grid containing the points of a structure that needs to be monitored. The technique used to identify the real scatterers from the given grid (which provide the main response and are not faded by layover) in a stack of refocused images is developed in the context of differential tomography [5]-[7]. This is obtained by exploiting the fact that each refocused scattering center will be at zero elevation in the local elevation-velocity (EV) plane. Thus, the actual detection consists in an elevation position testing relative to a certain grid.

To achieve an accuracy of the grid focusing in the centimeter range, a set of corrections are necessary regarding the atmospheric delays (for X-band the tropospheric delay can introduce a range error of around $3 \mathrm{~m}$, whereas the ionospheric delay is nonsignificant) and different offsets of the sensors (as described in [8] for TerraSAR-X products). An advantage of the refocusing approach on a specific grid is that no shifting or resampling (as part of the coregistration process) are needed because the samples get automatically aligned by refocusing each image on the same grid. Moreover, when computing the SAR impulse responses of the provided 3-D geographic model by refocusing the azimuth defocused data, the phase shifts corresponding to the acquisition geometry and to the variable Doppler centroid 
in the focused image (specific for sliding spotlight mode) are both taken into account. Hence, an implicit coregistration of the images is obtained and the interferometric phase can be computed as the phase difference of corresponding points. Notice that the proposed approach uses a provided digital elevation model (DEM) in a completely different manner than typical geometrical registration approaches working in the slant range domain. Furthermore, in comparison with the classical coregistered interferograms, a highly reflective scatterer with known coordinates that needs to be tracked (a mounted corner reflector, for instance) cannot have an inconvenient off-grid position for processing in the SAR image (e.g., to be at the edge of 4 neighboring pixels). It will always be placed in the center of the resolution cell on the new grid and its relative displacements will be accurately measured. The off-grid reflectors problem was also addressed in [9], where an algorithm for finding the reflectivity center is proposed. However, in the case of refocusing on a given grid, the purpose is to test if the scattering center is at the specified position (known with centimeter accuracy).

The proposed detection method based on elevation position testing could be also employed by converting the grid points from their original coordinates (e.g., Earth Centered Earth Fixed (ECEF) or latitude/longitude/altitude format) to the slant range-azimuth coordinates of a master image and interpolating the complex amplitude using the nearby pixels. However, compared with the refocusing approach, this interpolation-based method requires that all the images are first coregistered. This is even more relevant in the case of sliding spotlight images, where any interpolation required for coregistration has to be performed with a modulated kernel in order to follow the Doppler drift in azimuth [1]. From a different point of view, the refocusing procedure can be also seen as an equivalent interpolation kernel (considering that all operations involved are linear) whose parameters will be always adapted to the specific imaging mode. In addition, because the refocusing is applied to a small area of the initial SAR image the increased computational complexity should not be a significant drawback.

In the scattering centers identification procedure proposed in this paper, also the detection problem is posed in a different manner from classical approaches used in SAR tomography which are based on standard detection theory [6], [10]-[12]. Classically, the detection is done by comparing the normalized tomographic reconstructed peak to a certain threshold. In this case, the elevation dispersion inherently enters in the detection scheme by affecting the peak value. In the detection scheme proposed in this work the expected elevation is already known (zero for on-grid targets) and the detection actually consists in a position test of the overall maximum in the EV plane. Consequently, the elevation dispersion translates in the tolerated accuracy of the scattering center's position relative to the provided grid.

The refocusing-based detection and tracking technique is applied on a set of images acquired by the TerraSAR-X satellite over the Puylaurent dam in France between March-June 2012 and April-October 2013.

The remainder of this paper is organized as follows. Section II presents the proposed scattering centers monitoring algorithm and is divided in two parts. The azimuth defocusing

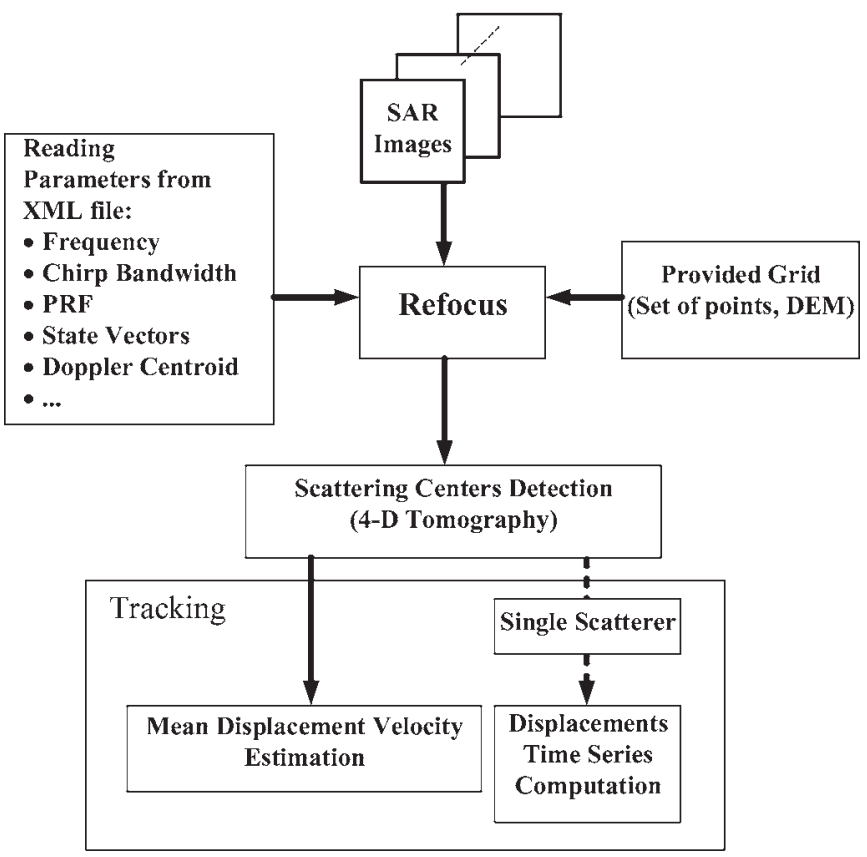

Fig. 1. Scattering centers detection and tracking based on SAR images refocusing.

is discussed first. Then, the refocusing on a given grid and the scattering center detection is described. In Section III a set of simulation results is presented in order to emphasize the performances and limitations of the proposed method particularly for a low number of satellite passes. Section IV shows the results of the algorithm applied to TerraSAR-X data. Afterward, Section $\mathrm{V}$ points out the main differences of the proposed approach relative to previous works. Finally, the conclusions are stated in Section VI.

\section{Scattering Centers Monitoring Procedure}

The scattering centers monitoring procedure is comprised of two stages: the refocusing of the acquired SAR images on the provided grid, followed by the scattering centers detection and displacements computation. A general diagram of the scattering centers detection and tracking procedure is displayed in Fig. 1. The refocusing algorithm consists in an azimuth defocusing of the initial SAR images followed by a focusing on the given grid, using a modified back-projection algorithm. The block diagram of the refocusing algorithm is shown in Fig. 2 and all the operations are described according to the flow on the processing chain. After the image is refocused, the scattering centers are detected and tracked using the differential tomography framework.

\section{A. Azimuth Defocusing}

The defocusing procedure is actually based on a reversed version of the Spectral Analysis (SPECAN) processing used for azimuth focusing [13], [14]. This approach is possible because the azimuth scaling consists only in Fourier transforms and complex multiplications that are reversible. The first operation consists in selecting from the initial image the slant 


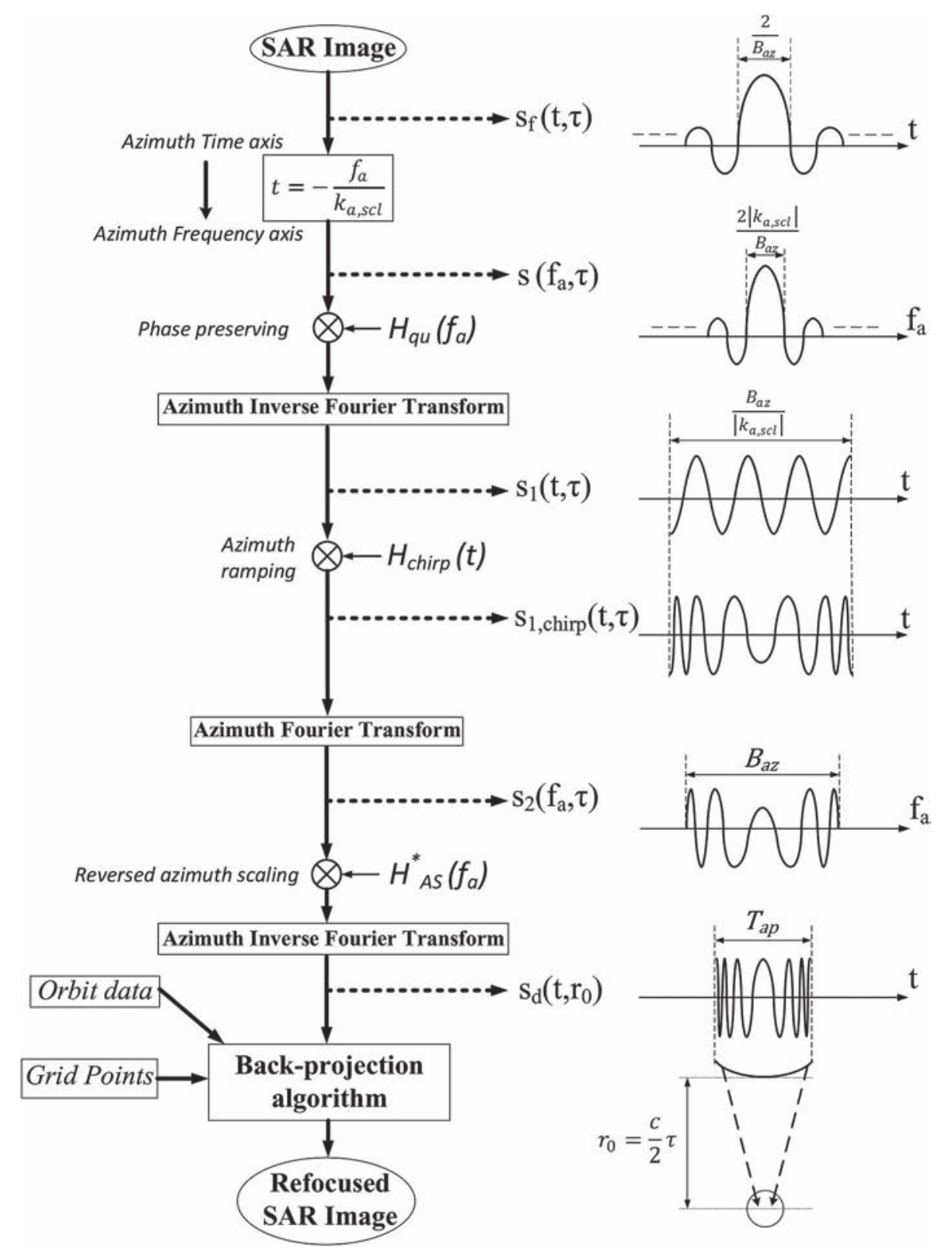

Fig. 2. Block diagram of the refocusing algorithm and qualitative representation of the real part of certain intermediate signals in the case of a single scatterer (zero Doppler centroid is assumed for representation simplicity).

range-azimuth region containing the target. Since the range compression is not modified during the processing, the selected region is cropped in range in order to reduce the computation time.

In the case of spotlight SAR images, the azimuth sampling frequency is larger than the raw data pulse repetition frequency (PRF) in order to cover the complete spotlight bandwidth. In the defocusing procedure, the sampling frequency is the one of the SAR image; thus, the subaperture approach used for realtime focusing [15] does not need to be employed. Therefore, the subaperture recombination from the focusing algorithm in [14] can be skipped (it was necessary only to keep the PRF at the value from the raw data acquisition just until the final azimuth Fourier transform). An advantage of processing the entire aperture is that the average Doppler centroid will have a very small value that minimizes the azimuth time shifts caused by the azimuth scaling.

The focused 2-D signal for $N$ scatterers (equal to the number of pixels of the cropped SAR image) can be written as

$$
\begin{aligned}
s_{f}(t, \tau)= & \sum_{i=1}^{N} A_{i} \exp \left(-j 2 \pi f_{c} \tau\right) \operatorname{sinc}\left[\pi B_{r}\left(\tau-\tau_{i}\right)\right] \\
& \times \operatorname{sinc}\left[\pi B_{a z}\left(t-t_{i}\right)\right] \exp \left(j 2 \pi f_{\mathrm{DC}}\left(t_{i}\right)\left(t-t_{i}\right)\right)
\end{aligned}
$$

where $t$ is the azimuth (slow) time axis, $\tau$ is the slant range (fast) time axis, $B_{a z}$ is the target azimuth bandwidth, $B_{r}$ is the range chirp bandwidth, $f_{c}$ is the central frequency, $A_{i}$, $t_{i}, \tau_{i}$ represent respectively the complex amplitude, the zero 
Doppler azimuth time and the slant range delay at closest approach for the scatterer $i$ and $f_{\mathrm{DC}}\left(t_{i}\right)$ is the Doppler centroid corresponding to a target at the zero Doppler azimuth time $t_{i}$. For simplicity, rectangular windows for both range and azimuth data are assumed, hence the sinc functions in (1). Notice that this analytic expression takes into account that in sliding spotlight mode the spectrum of each target in the focused image is centered around the Doppler centroid corresponding to the target's azimuth position [16], [17]. Generally, the Doppler centroid can be written as a linear function of the zero Doppler azimuth time

$$
f_{\mathrm{DC}}(t)=f_{\mathrm{DC}, 0}+\alpha t
$$

where $f_{\mathrm{DC}, 0}$ is the Doppler centroid at $t=0$ and $\alpha$ is the Doppler drift rate in the focused scene caused by the beam sweeping in spotlight acquisition modes.

In the SPECAN processing [18] each zero Doppler azimuth time from the focused image is actually linked to an azimuth frequency $f_{a}$ by the scaling Doppler rate $k_{a, s c l}$

$$
t=-\frac{f_{a}}{k_{a, s c l}}
$$

and the image can be regarded as a function of azimuth frequency and fast time

$$
\begin{aligned}
s\left(f_{a}, \tau\right)= & s_{f}\left(-\frac{f_{a}}{k_{a, s c l}}, \tau\right)=\sum_{i=1}^{N} A_{i} \exp \left(-j 2 \pi f_{c} \tau\right) \\
& \times \operatorname{sinc}\left[\pi B_{r}\left(\tau-\tau_{i}\right)\right] \operatorname{sinc}\left[\pi \frac{B_{a z}}{k_{a, s c l}}\left(f_{a}+k_{a, s c l} t_{i}\right)\right] \\
& \times \exp \left(-j 2 \pi \frac{f_{\mathrm{DC}}\left(t_{i}\right)}{k_{a, s c l}}\left(f_{a}+k_{a, s c l} t_{i}\right)\right)
\end{aligned}
$$

The first multiplication in the processing is made for phase preserving given the subsequent processing steps and is described by a quadratic phase term

$$
H_{q u}\left(f_{a}\right)=\exp \left(j \pi \frac{f_{a}^{2}}{k_{a, s c l}}\right) .
$$

Next an azimuth inverse Fourier transform is applied and leads to a 2-D signal in the azimuth-fast time domain (computed analytically in a similar fashion as in [19] for the residual video phase removal)

$$
\begin{aligned}
s_{1}(t, \tau)= & C_{1} \sum_{i=1}^{N} A_{i} \exp \left(-j 2 \pi f_{c} \tau\right) \operatorname{sinc}\left[\pi B_{r}\left(\tau-\tau_{i}\right)\right] \\
& \times \operatorname{rect}\left[\frac{k_{a, s c l}}{B_{a z}}\left(t-t_{i}-\frac{f_{\mathrm{DC}}\left(t_{i}\right)}{k_{a, s c l}}\right)\right] \\
& \times \exp \left(j \pi k_{a, s c l}\left(-2 t_{i} t+t_{i}{ }^{2}\right)\right) .
\end{aligned}
$$

By chirping $s_{1}(t, \tau)$ with the function

$$
H_{\text {chirp }}=\exp \left(j \pi k_{a, s c l} t^{2}\right)
$$

the resulting signal becomes a sum of chirp functions in azimuth having the zero frequency points at the closest approach azimuth times of each target

$$
\begin{aligned}
& s_{1, \operatorname{chirp}}(t, \tau)=C_{1} \sum_{i=1}^{N} A_{i} \exp \left(-j 2 \pi f_{c} \tau\right) \operatorname{sinc}\left[\pi B_{r}\left(\tau-\tau_{i}\right)\right] \\
& \times \operatorname{rect}\left[\frac{k_{a, s c l}}{B_{a z}}\left(t-t_{i}-\frac{f_{\mathrm{DC}}\left(t_{i}\right)}{k_{a, s c l}}\right)\right] \exp \left[j \pi k_{a, s c l}\left(t-t_{i}\right)^{2}\right] .
\end{aligned}
$$

In the next step an azimuth Fourier transform is applied to the signal in (8) using the principle of stationary phase (PSP) [19], [20]. The PSP can be employed because the signal is a sum of chirp functions each of which bringing one stationary point in the computation of the Fourier integral for every azimuth frequency. The result is written as

$s_{2}\left(f_{a}, \tau\right)=C_{2} \sum_{i=1}^{N} A_{i} \exp \left(-j 2 \pi f_{c} \tau\right) \operatorname{sinc}\left[\pi B_{r}\left(\tau-\tau_{i}\right)\right]$

$\times \operatorname{rect}\left[\frac{f_{a}-f_{\mathrm{DC}}\left(t_{i}\right)}{B_{a z}}\right] \exp \left(-j \pi \frac{f_{a}{ }^{2}}{k_{a, s c l}}\right) \exp \left(-j 2 \pi f_{a} t_{i}\right)$.

The signal in (9) is multiplied with a complex conjugated version of the azimuth scaling function [14] expressed as

$$
\begin{aligned}
& H_{A S}^{*}\left(f_{a}, \tau\right)=\exp \left(j \pi \frac{f_{a}^{2}}{k_{a, s c l}}\right) \\
& \times \exp \left[-j 2 \pi f_{c} \tau\left(\sqrt{1-\left(\frac{f_{a} \lambda}{2 v_{0}}\right)^{2}}-1\right)\right]
\end{aligned}
$$

where $\lambda$ is the wavelength at central frequency, and $v_{0}$ is the zero Doppler azimuth velocity. This operation converts the chirp's quadratic phase to the original range dependent (through the fast time $\tau$ ) hyperbolic phase history. The signal takes the form

$$
\begin{aligned}
s_{2, \text { hyp }}\left(f_{a}, \tau\right)= & C_{2} \sum_{i=1}^{N} A_{i} \exp \left(-j 2 \pi f_{c} \tau\right) \operatorname{sinc}\left[\pi B_{r}\left(\tau-\tau_{i}\right)\right] \\
& \times \operatorname{rect}\left[\frac{f_{a}-f_{\mathrm{DC}}\left(t_{i}\right)}{B_{a z}}\right] \exp \left(-j 2 \pi f_{a} t_{i}\right) \\
& \times \exp \left[-j 2 \pi f_{c} \tau\left(\sqrt{1-\left(\frac{f_{a} \lambda}{2 v_{0}}\right)^{2}}-1\right)\right] .
\end{aligned}
$$

The last step is an azimuth inverse Fourier transform computed for each term of the sum in (11) using the Fourier transform pair 
in [21]. After this transform the azimuth defocused 2-D signal has the form

$$
\begin{aligned}
s_{d}\left(t, r_{0}\right)= & C \sum_{i=1}^{N} A_{i} \operatorname{sinc}\left[\frac{2 \pi B_{r}}{c}\left(r_{0}-r_{0, i}\right)\right] \\
& \times \operatorname{rect}\left[\frac{t-\left(t_{i}-\frac{T_{a p}}{B_{a z}} f_{\mathrm{DC}}\left(t_{i}\right)\right)}{T_{a p}}\right] \\
& \times \exp \left(-j \frac{4 \pi}{\lambda} \sqrt{r_{0}^{2}+\left[v_{0}\left(t-t_{i}\right)\right]^{2}}\right)
\end{aligned}
$$

where $c$ is the speed of light and $T_{a p}$ is the equivalent synthetic aperture (illumination) time [1]. In (12) each fast time $\tau$ was written as $2 r_{0} / c$, where $r_{0}$ is the corresponding closest approach distance.

Given the support of the rectangular window in (12) and that for a SAR image with azimuth extent $t_{\text {scene }}$ the closest approach azimuth times can vary between $-t_{\text {scene }} / 2$ and $t_{\text {scene }} / 2$ the minimum necessary azimuth support is

$$
T_{a z, \min }=t_{\text {scene }}\left(1-\alpha \frac{T_{a p}}{B_{a z}}\right)+T_{a p} .
$$

Hence, the number of points in azimuth should respect the condition

$$
N_{a z} \geq F_{s} T_{a z, \min }
$$

where $F_{s}$ is the azimuth sampling frequency (the equivalent PRF). The necessary number of azimuth points can eventually be chosen as the next power of 2 which fulfills (14) and is obtained by zero padding the initial data. Having $N_{a z}$ and taking into account (3), the scaling Doppler rate is given by

$$
k_{a, s c l}=-\frac{F_{s}{ }^{2}}{N_{a z}} .
$$

\section{B. Grid Focusing}

Notice that the signal in (12) has the natural hyperbolic phase history for each target and is not affected by range migration. The discrete azimuth defocused signal can be written as $s_{d}[m, n]=s_{d}(m \delta t, n \delta r)$, where $\delta t=1 / F_{s}$ is the azimuth time spacing and $\delta r$ the range spacing. Seen as a matrix, $s_{d}[m, n]$ has on each column the phase history for a certain closest approach slant range and each line contains a range profile.

The grid focusing procedure starts by extracting the annotated orbit data [22]. The envisaged geometry is shown in Fig. 3(a). The unit vector $\overrightarrow{\boldsymbol{u}}$ of the azimuth direction is computed as the normalized velocity vector of the satellite at the azimuth time of the image center. For an azimuth resolution larger than $1 \mathrm{~m}$ the straight line trajectory approximation is satisfactory (the curved orbit correction is needed only for staring spotlight mode [23]). The position of the satellite's antenna phase center (APC) at a given azimuth time $t$ can be written as

$$
\overrightarrow{\boldsymbol{r}}_{\boldsymbol{a}}(t)=\overrightarrow{\boldsymbol{r}}_{\boldsymbol{a}, \mathbf{0}}+v_{0} t \overrightarrow{\boldsymbol{u}}
$$

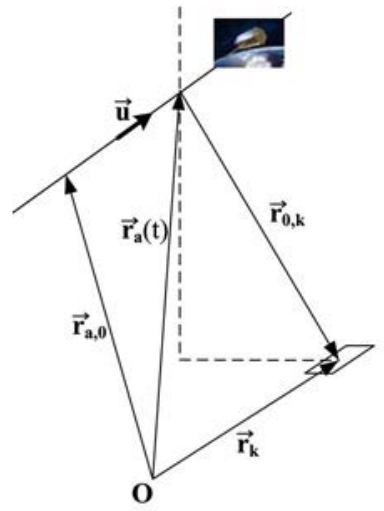

(a) (b)

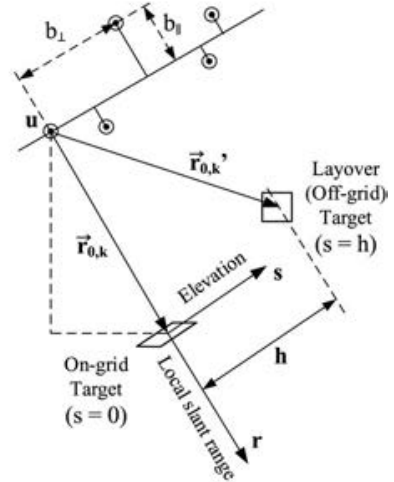

Fig. 3. (a) Refocusing geometry for a target with a known on-grid position $\vec{r}_{\boldsymbol{k}}$. (b) Layover detection geometry based on zero elevation for on-grid targets.

where $\overrightarrow{\boldsymbol{r}}_{\boldsymbol{a}, 0}$ is the APC position vector at $t=0$. For a given target having the position vector $\vec{r}_{k}$, the closest approach distance to the synthetic aperture is given by

$$
r_{0, k}=\left\|\left(\overrightarrow{\boldsymbol{r}}_{\boldsymbol{a}, \mathbf{0}}-\overrightarrow{\boldsymbol{r}}_{\boldsymbol{k}}\right)-\left[\left(\overrightarrow{\boldsymbol{r}}_{\boldsymbol{a}, \mathbf{0}}-\overrightarrow{\boldsymbol{r}}_{\boldsymbol{k}}\right) \cdot \overrightarrow{\boldsymbol{u}}\right] \overrightarrow{\boldsymbol{u}}\right\| .
$$

The response of this target is computed using a time-domain back-projection algorithm (matched filtering based technique) [24]-[27] modified for data with no range cell migration having the phase history of a point target on a single column of the matrix $s_{d}[m, n]$. Hence, the target response is computed as

$g\left(\overrightarrow{\boldsymbol{r}}_{\boldsymbol{k}}\right)=\sum_{m=M_{-}}^{M_{+}} s_{d}\left(m \delta t, r_{0, k}\right) \exp \left(j \frac{4 \pi f_{c}}{c}\left\|\overrightarrow{\boldsymbol{r}}_{\boldsymbol{a}}(m \delta t)-\overrightarrow{\boldsymbol{r}}_{\boldsymbol{k}}\right\|\right)$

where $M_{-}$and $M_{+}$are the limits corresponding to the total illumination duration of the respective target (the equivalent synthetic aperture time $T_{a p}$ ). The zero Doppler azimuth time for the target placed at $\overrightarrow{\boldsymbol{r}}_{\boldsymbol{k}}$ is expressed as

$$
t_{\overrightarrow{\boldsymbol{r}}_{\boldsymbol{k}}}=\left(\overrightarrow{\boldsymbol{r}}_{\boldsymbol{k}}-\overrightarrow{\boldsymbol{r}}_{\boldsymbol{a}, \mathbf{0}}\right) \cdot \overrightarrow{\boldsymbol{u}} / v_{0}
$$

and the summation limits are

$$
M_{ \pm}=\left[F_{s}\left(t_{\overrightarrow{\boldsymbol{r}}_{k}}-\frac{T_{a p}}{B_{a z}} f_{\mathrm{DC}}\left(t_{\overrightarrow{\boldsymbol{r}}_{\boldsymbol{k}}}\right) \pm \frac{T_{a p}}{2}\right)\right] .
$$

Notice that $r_{0, k}$ in (18) is not necessarily on the range grid (is not written as $n \delta r$ ) and hence the value of $s_{d}\left(m \delta t, r_{0, k}\right)$ is interpolated in the algorithm from the matrix $s_{d}[m, n]$. The refocusing is implemented by applying (18) to each point from the given set.

\section{Detection and Tracking of Scattering Centers}

For two refocused images, the stable scatterers could be detected by classical coherence evaluation on a vicinity of each refocused point. However, in order to determine if the reflecting scattering center is actually at the given point an approach based on a series of acquisitions is needed in order to create an elevation aperture. Considering the 4-D SAR imaging model 


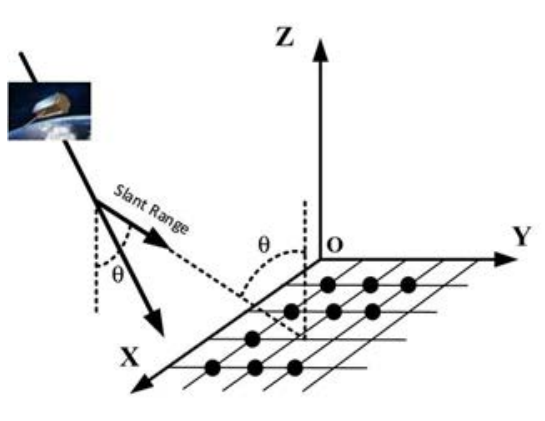

(a)

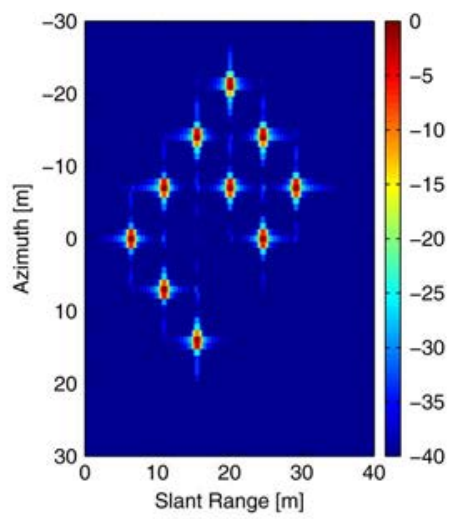

(b)

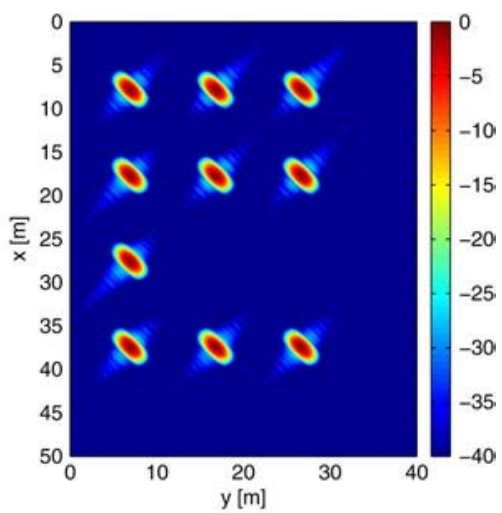

(c)

Fig. 4. Refocusing Algorithm Simulation. (a) Simulation scenario. (b) Focused SAR image with the Frequency Scaling Algorithm in slant range-azimuth geometry. (c) Refocused image on a grid placed in the horizontal plane containing the scatterers.

in [6] for each scatterer $k$ from the given grid (on-grid target situated at $\overrightarrow{\boldsymbol{r}}_{\boldsymbol{k}}$ ) the received signal vector is written as

$$
\boldsymbol{g}\left(\overrightarrow{\boldsymbol{r}}_{\boldsymbol{k}}\right)=\int_{\Delta s} \int_{\Delta v} p_{\gamma}(s, v) \mathbf{a}(s, v) d s d v
$$

where $p_{\gamma}(s, v)=\gamma(s) p(s, v)$, with $\gamma(s)$ being the reflectivity profile along elevation $s$ and $p(s, v)$ the EV spectral distribution of the displacement terms. $\Delta s$ and $\Delta v$ are the elevation and velocity supports of $p_{\gamma}(s, v)$ and $\mathbf{a}(s, v)$ is the steering vector, whose elements are defined as

$$
a_{n}(s, v)=\exp \left[j 2 \pi\left(\frac{2 b_{\perp, n}}{\lambda r_{0, k}} s+\frac{2 t_{n}}{\lambda} v\right)\right] .
$$

In (22), $b_{\perp, n}$ is the orthogonal baseline computed for the target $k$ (perpendicular to $\overrightarrow{\boldsymbol{r}}_{\mathbf{0}, \boldsymbol{k}}$ ) relative to the first acquisition, $t_{n}$ is the acquisition time and $\lambda$ is the central wavelength. Because the $\left(b_{\perp, n}, t_{n}\right)$ pairs are sparse and nonuniform, the function $p_{\gamma}(s, v)$ is reconstructed using the Capon filter [7], [28]

$$
\hat{p_{\gamma}}(s, v)=\frac{\mathbf{a}^{H}(s, v) \hat{\mathbf{R}}^{-1} \boldsymbol{g}\left(\overrightarrow{\boldsymbol{r}}_{\boldsymbol{k}}\right)}{\mathbf{a}^{H}(s, v) \hat{\mathbf{R}}^{-1} \mathbf{a}(s, v)}
$$

where $\hat{\mathbf{R}}$ is a multilook estimate of the data vector $\boldsymbol{g}\left(\overrightarrow{\boldsymbol{r}}_{\boldsymbol{k}}\right)$ covariance matrix. The power spectral density (PSD) is then obtained as the power of $\hat{p_{\gamma}}(s, v)$. By changing the geometrical $\mathrm{EV}$ configuration for each refocused target, its position on the elevation axis is at $s=0$ and any other scatterer situated in layover will be at another distance as presented in Fig. 3(b). Therefore, the persistent scatterers from the set of targets which have the real scattering center at the given position and are not affected by layover are the ones that have only one significant maximum value of the PSD in the EV plane at zero elevation clearly separated from other eventual local peaks corresponding to layover targets. Moreover, the dispersion of the elevation estimator can be interpreted as the positioning accuracy of the detected scatterer relative to the provided grid. The mean displacement velocity (MDV) $\overline{v_{0}}$ of a detected scatterer is estimated as the location of the peak on the velocity axis at zero elevation.
The displacements time series $d\left(t_{n}\right)$ of a detected scatterer can be viewed as a sum between the linear displacement and the residual unmodeled motion. Hence, for a single scatterer at zero elevation, not influenced by layover targets the time series is expressed as [6]

$$
d\left(t_{n}\right)=\overline{v_{0}} t_{n}+\frac{\lambda}{4 \pi} \arg \left\{g_{n}\left(\vec{r}_{k}\right) \exp \left(-j \frac{4 \pi \overline{v_{0}} t_{n}}{\lambda}\right)\right\} .
$$

This expression is similar to the persistent scatterer interferometry (PSI) case except that here the linear displacement phase term is first subtracted. By computing the time series in this way, only the nonlinear displacement relative to the linear trend has to be below half-wavelength and not the whole displacement.

\section{Simulation Results}

\section{A. Refocusing Algorithm Simulations}

Here, simulation results are presented in order to test the proposed refocusing algorithm and to compare its performances to other methods of conversion to ground geometry.

1) Radar Geometry to Ground Geometry: A scene containing 10 point-like scatterers arranged in a rectangular grid, as shown in Fig. 4(a) is simulated. The distance between two neighboring points of the grid is $10 \mathrm{~m}$ on $\mathrm{Ox}$ and $\mathrm{Oy}$. The flight path is contained in a horizontal plane (parallel to xOy) and oriented at $45^{\circ}$ with respect to Ox. The simulation parameters are picked according to the typical values for the TerraSAR-X satellite operating in high-resolution spotlight mode: central frequency $9.65 \mathrm{GHz}$, total azimuth bandwidth $7 \mathrm{kHz}$, azimuth sampling frequency $8.5 \mathrm{kHz}$, equivalent synthetic aperture duration $1.5 \mathrm{~s}$, zero Doppler velocity $7 \mathrm{~km} / \mathrm{s}$, mean slant range $750 \mathrm{~km}$, incidence angle $40^{\circ}$. The dechirp-on-receive response of the targets is computed for every pulse in zero Doppler coordinates considering the stop and go approximation. The SAR image is focused using the Frequency Scaling Algorithm [14] with Hamming window weighing in both slant range and azimuth. Fig. 4(b) shows the obtained image in slant range-azimuth geometry. The proposed refocusing algorithm is applied on this image using as new grid an oversampled version 


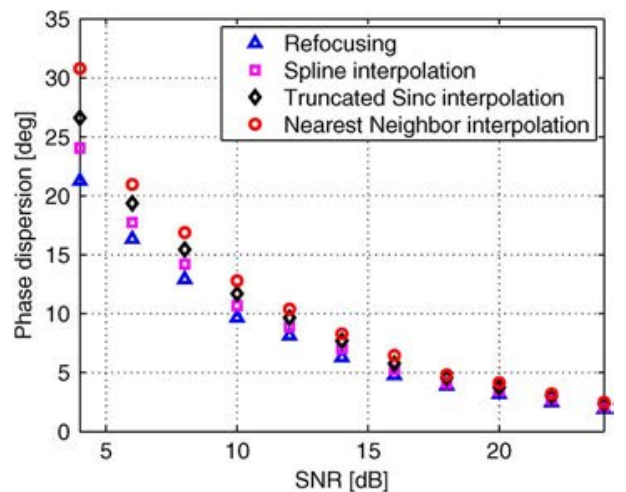

(a)

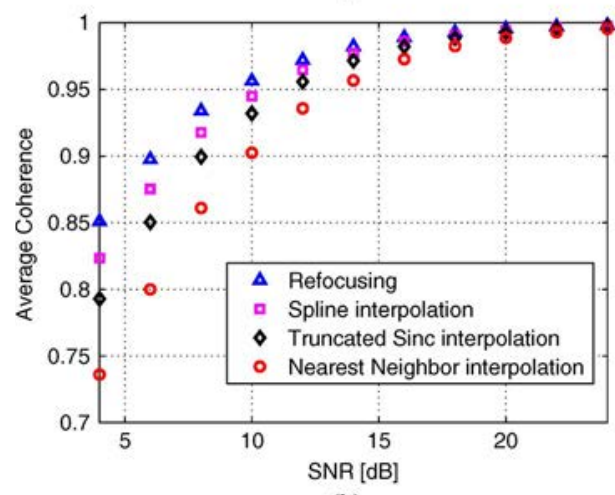

(b)

Fig. 5. Comparison between the refocusing approach and 3 interpolationbased methods. (a) Phase dispersion. (b) Average coherence.

of the rectangular grid on which the scatterers are placed having a $20 \mathrm{~cm}$ distance between neighboring points on both axis. The refocused image is presented in Fig. 4(c). Notice that in the initial image, the scatterers are disposed in a parallelogram shape due to the slant range geometry, whereas in the image refocused on the horizontal grid, the correct rectangular shape appears.

2) Refocusing Approach Versus Grid Interpolation: The proposed position test detection method could also be carried out if the provided 3-D model would be converted to slant range-azimuth coordinates and the SAR impulse responses interpolated using nearby pixels. Assuming that the images from the acquired data set are coregistered, the interpolation of the SAR impulse response can be done by various methods.

This section presents a few simulation results aimed to compare the performances of the refocusing approach with three usual interpolation methods: spline-based interpolation, truncated sinc and nearest neighbor. The scenario considered for this simulation involves a target placed at random positions on the ground such that in some cases is in the center of a pixel and in others at the edge of 4 neighboring pixels. The refocusing/interpolation grid is a rectangular one centered each time at the target's position. The performances of the methods are compared in terms of phase dispersion and average coherence. One thousand realizations are simulated for different values of the SNR. Fig. 5 shows the phase dispersion and average coherence for various signal-to-noise (SNR) ratios. The refocusing approach provides better results particularly for low

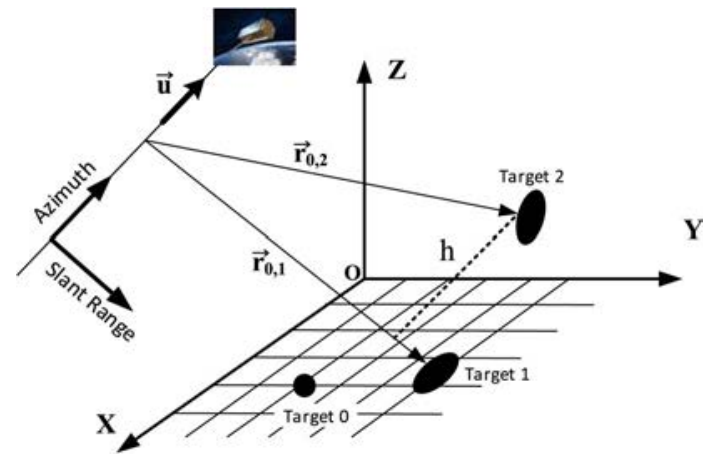

Fig. 6. Envisaged geometric configuration for the detection and tracking simulations.

TABLE I

FALSE ALARM PRoBAbility (\%)

\begin{tabular}{|c||c|c|c|}
\hline Accuracy & 5 & 8 & 15 \\
\hline \hline $0.5 \mathrm{~m}$ & 0.74 & 0.60 & 0.46 \\
\hline $2.5 \mathrm{~m}$ & 2.61 & 2.48 & 1.54 \\
\hline $5 \mathrm{~m}$ & 5.09 & 4.95 & 3.01 \\
\hline
\end{tabular}

SNR values, whereas for high SNRs the methods are comparable. These results are linked to the fact that the refocusing is each time adapted to the specific imaging parameters.

\section{B. Scattering Centers Monitoring Simulations}

This section presents the results of a set of simulations that aim to highlight the performances and limitations of the proposed scattering centers monitoring procedure. The impact of different configurations/parameters on the detection and tracking of a scatterer at a certain grid position is studied. The conducted simulations want to emphasize the behavior of the algorithm particularly for a relatively small number of available satellite passes (which can be a practical problem in shortterm infrastructure monitoring). Obviously the simulated cases are not exhaustive, but they can give an idea of the expected performances in different scenarios.

The main settings used in the simulations are the same as those presented in Section III-A1, but the simulated scene is the one from Fig. 6. Target 1 is a scattering region obtained from the superposition of a few point-like targets which are all displaced in the same manner in every test. Target 0 is used as reference point and all the computed displacements are relative to it. Target 2 is positioned such that it is in layover with target 1 , but its assigned amplitude is nonzero only for the simulations involving layover scenarios. Several scenes (corresponding to successive satellite tracks) like the one in Fig. 6 are simulated by computing the refocused image on a grid in the $\mathrm{xOy}$ plane in an area near target 1 . For each track, target 1 is displaced according to the considered test case and the baseline is arbitrarily chosen in keeping with the $\pm 250 \mathrm{~m}$ orbit tube of the TerraSAR-X satellite. In each simulation are studied the effects of the number of tracks, the SNR and the presence of one or two scatterers in elevation on parameters like detection probability and velocity/displacement bias and dispersion. The 


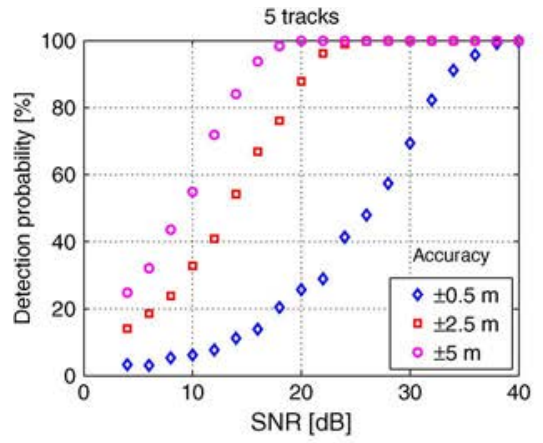

(a)

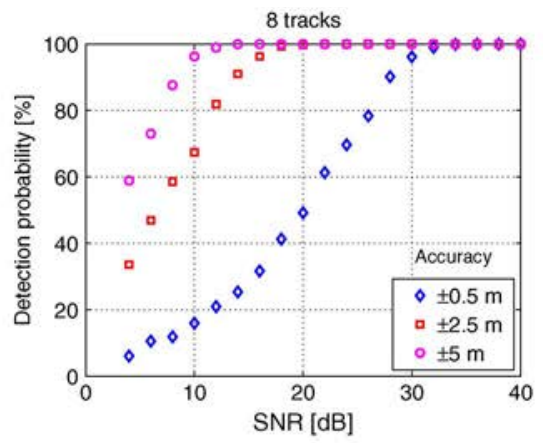

(b)

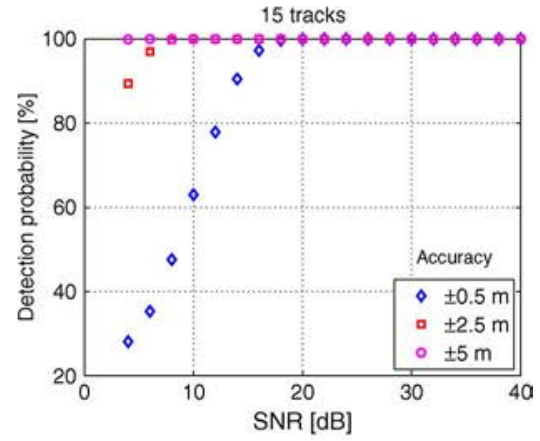

(c)

Fig. 7. Detection probability versus SNR for one scatterer having a linear motion in LOS. The considered accuracies are $0.5 \mathrm{~m}, 2.5 \mathrm{~m}$, and $5 \mathrm{~m}$ and the number of simulated satellite passes are (a) 5 tracks, (b) 8 tracks, and (c) 15 tracks.

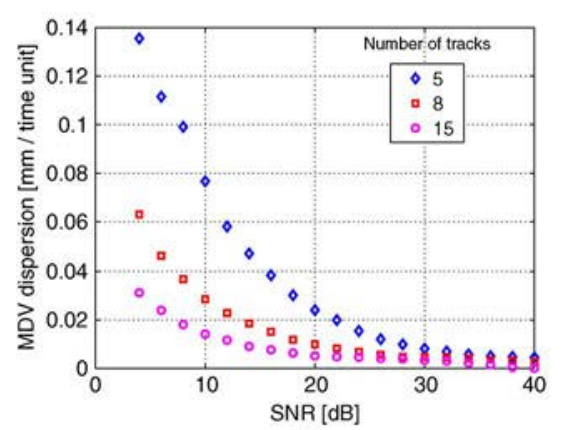

(a)

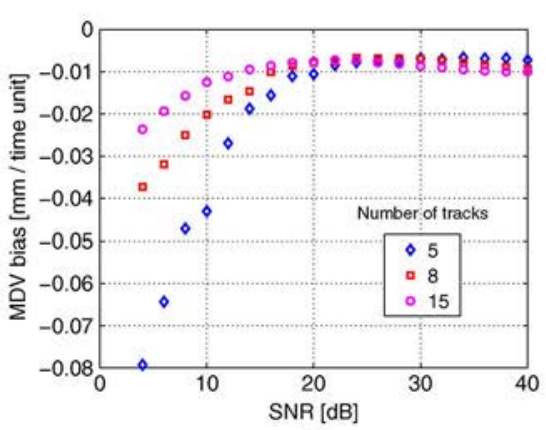

(b)

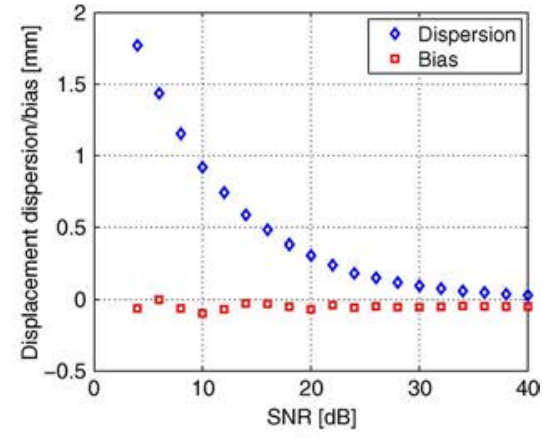

(c)

Fig. 8. (a) Dispersion of estimated MDV, (b) bias of estimated MDV, and (c) displacement dispersion/bias versus SNR for one scatterer with linear motion in LOS.

noise added to obtain a certain SNR leads to a circular Gaussian complex amplitude fluctuating from one look to another. For each SNR value, 1000 realizations of the process are simulated.

The covariance matrix was estimated using the sample covariance matrix estimator [29]. The number of neighboring points for the estimation was chosen as a compromise between the degradation in resolution and the condition to have a positive definite estimated matrix (the number of looks used for averaging to be at least equal to the number of tracks [5]). Therefore, the number of looks used in each case was the number of tracks plus one.

As described in the previous section, the detection of a scatterer at a given position relies on the presence of the highest peak in the EV plane at zero elevation. From a practical standpoint, this verification means to test on the 2-D EV grid if the maximum appears at one of the zero elevation bins. In the presence of noise the maximum can very likely appear in a bin near zero elevation depending on the grid's elevation step. Consequently, a tolerance should be added to the detection criteria related to the detection probability. This issue will also impose the minimum necessary elevation step of the EV grid (the maximum value is given by the Nyquist resolution divided by a possible over-sampling factor). Given these matters, the detection criteria is reformulated as follows: a given point is a scattering center if the maximum in the EV plane is placed in the interval $[-\delta s,+\delta s]$, where $\delta s$ is the detection accuracy. Notice that the detection accuracy is a positioning accuracy and is not related to the resolution in elevation. The elevation step of the grid can be picked as high as $2 \delta s$ for computational ease, whereas the velocity step should be chosen as small as possible for an accurate estimate of the displacement velocity (in the simulations was chosen $0.01 \mathrm{~mm} /$ time unit). The notion of detection probability used hereafter is defined as the number of detections of a scattering center in the interval $[-\delta s,+\delta s]$ divided by the total number of realizations. In a similar manner, the false alarm probability can be viewed as the number of realizations for which the maximum value appears in the interval $[-\delta s,+\delta s]$ divided by the total number of realizations when the data vector $\boldsymbol{g}\left(\vec{r}_{\boldsymbol{k}}\right)$ contains only noise. In the conducted analysis, we considered the detection accuracies $0.5 \mathrm{~m}, 2.5 \mathrm{~m}$, and $5 \mathrm{~m}$ and the number of tracks was 5, 8, and 15 . For each combination, we have determined by simulation the resulting false alarm probability, which is presented in Table I. Notice that for a given set of acquisitions, the tolerated accuracy imposes a fixed level of the false alarm probability.

The results of various simulation configurations are presented in the following.

1) One Scatterer, Linear Motion: The first case studied is the one where there is only one scatterer, which has a linear motion in line of sight (LOS). The detection probability curves versus SNR for different accuracies and number of tracks are plotted in Fig. 7. Fig. 8(a) shows the dispersion of the estimated MDV and Fig. 8(b) the MDV's bias. Naturally, the detection probability and MDV bias/dispersion enhance as 


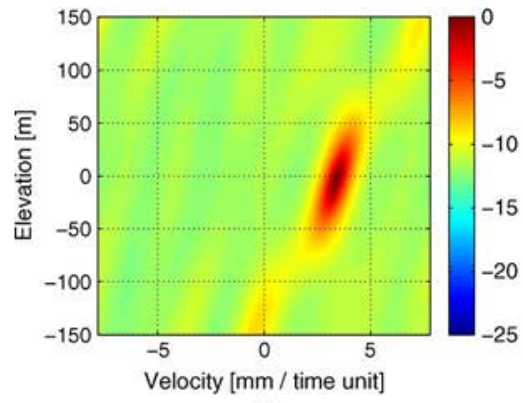

(a)

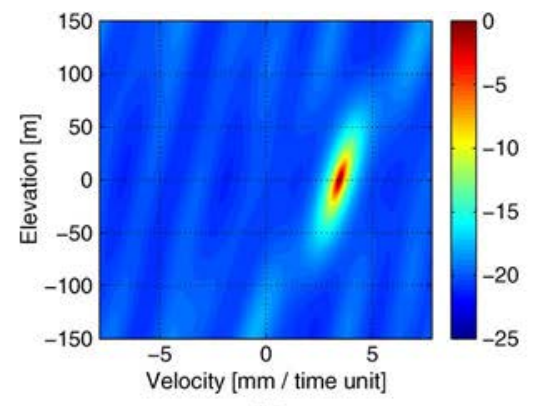

(b)

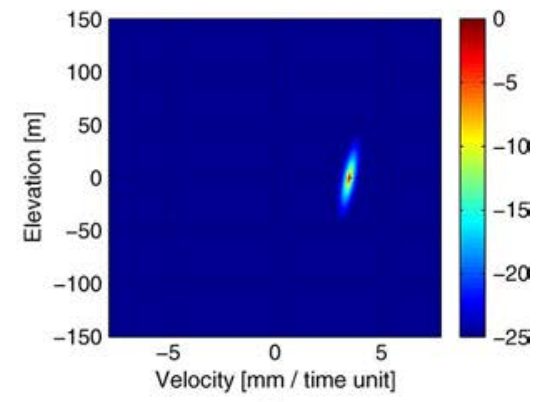

(c)

Fig. 9. Estimated EV PSD planes for eight tracks and different SNRs (a) $5 \mathrm{~dB}$, (b) $15 \mathrm{~dB}$, and (c) $25 \mathrm{~dB}$.

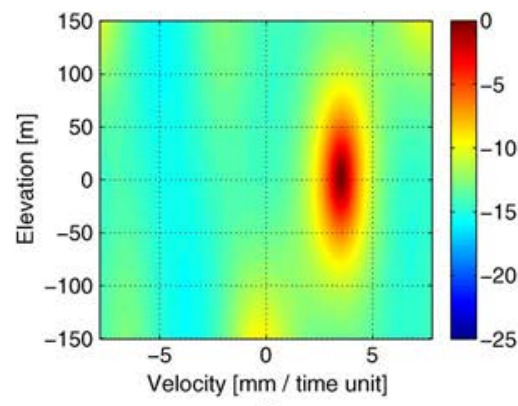

(a)

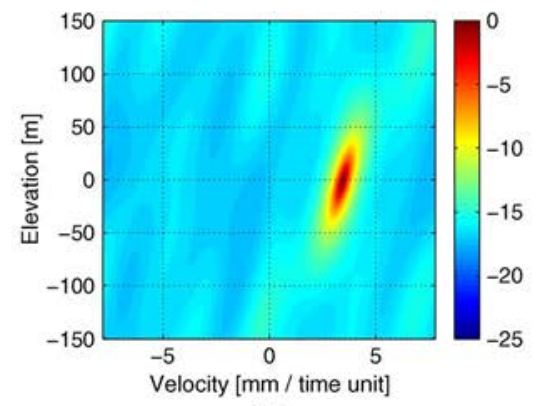

(b)

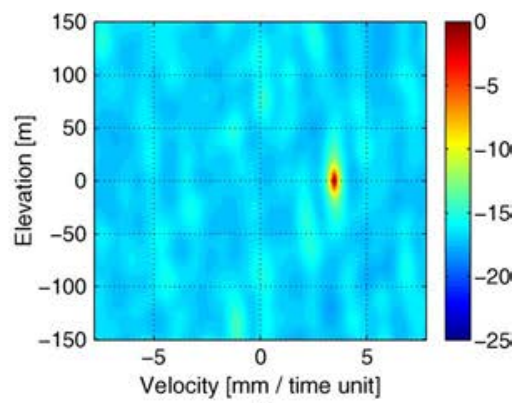

(c)

Fig. 10. Estimated EV PSD planes for a SNR of $10 \mathrm{~dB}$ and different number of satellite passes (a) 5 tracks, (b) 8 tracks, and (c) 15 tracks.

the number of tracks increases. The dispersion and bias of the computed displacements using (24) is presented in Fig. 8(c). The estimated EV PSD planes for various SNRs and number of tracks are presented in Figs. 9 and 10, respectively. As expected, the noise floor of the PSD in the estimated EV plane is higher for low SNRs and the main scatterer's lobe diminishes as the number of tracks increases.

2) One Scatterer, Linear Trend and Unmodeled Nonlinear Motion: For this part, unmodeled nonlinear motion is added to the scatterer simulated in the previous case. The additional motion consists in randomly generated sets of displacements having different dispersions. In each case, the mean of the nonlinear motion is subtracted in order to maintain the mean velocity of the linear motion. Fig. 11 shows the impact of this change on the detection probability (using \pm 2.5 -m accuracy) and MDV bias/dispersion. The curves plotted for 0 -mm dispersion correspond to the pure linear motion discussed in the previous section. Notice in Fig. 11(a) and (b) that the detection probability is highly diminished by the unmodeled motion for the 5 tracks case, whereas for 8 satellite passes, the effect is negligible. For a higher number of tracks the impact on the detection probability is similar to the 8 tracks situation. Regarding the MDV dispersion and bias shown in Fig. 11(c) and (d), in addition to an additional small bias the effect of the nonlinear motion is insignificant provided that the necessary number of tracks for a good detection probability is available.

The dispersion and bias for the displacements time series computed like in the PSI-case are not affected by the nonlinear motion. This happens because for the displacement computation, only one track is used relative to the reference one (different from the EV-plane which is estimated using all acqui- sitions). Hence, the dispersion/bias plots are actually the same as for pure linear motion [Fig. 8(c)].

3) Two Scatterers in Layover, Linear Motion: In this scenario, as shown in Fig. 6, a second scatterer (target 2) is placed in layover with the one of interest (target 1). The effect of the second scatterer on the detection and tracking of the main target is investigated for different amplitudes of target 2 (relative to target 1). Fig. 12 shows the simulation outcome for the two scatterers case. The detection results for small number of tracks are very sensitive to the presence of the other scatterer and consequently the tracking results are presented only for the 15 passes case. Fig. 13 shows the estimated PSD in the EV planes (at $20 \mathrm{~dB}$ SNR) for different relative amplitudes. Notice that the second scatterer fades when its amplitude decreases, but the EV plane noise floor gets quite high in its presence and taking into account the EV planes aspect from the single scatterer cases this happens mainly due to side-lobes interaction between the two scatterers. Therefore, a pertinent criteria for the single scatterers identification is to have only one significant peak at zero elevation and any other local maximum to be much lower than it (e.g., a value of $10 \mathrm{~dB}$ is appropriate in the simulated cases).

\section{EXPERIMENTAL RESUlTS}

The proposed monitoring algorithm was tested on two sets of 8 high-resolution sliding spotlight images acquired with the TerraSAR-X satellite over the Puylaurent dam in France between March-June 2012 and April-October 2013, respectively.

Depending on the parameters estimated by the SAR processor (e.g., Doppler centroid and Doppler drift rate), the TerraSAR-X sliding spotlight SAR images may have slightly 


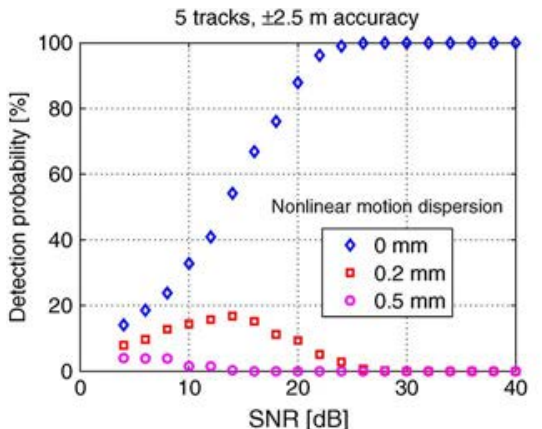

(a)

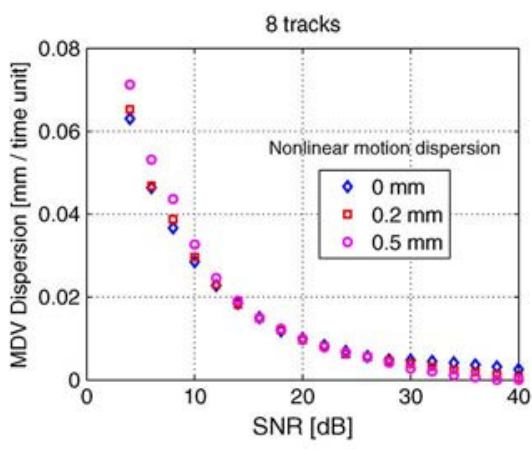

(c)

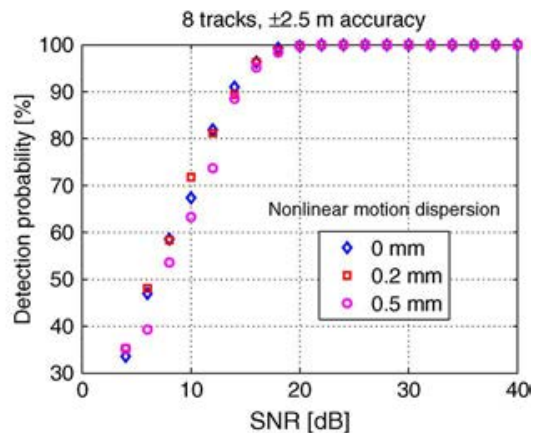

(b)

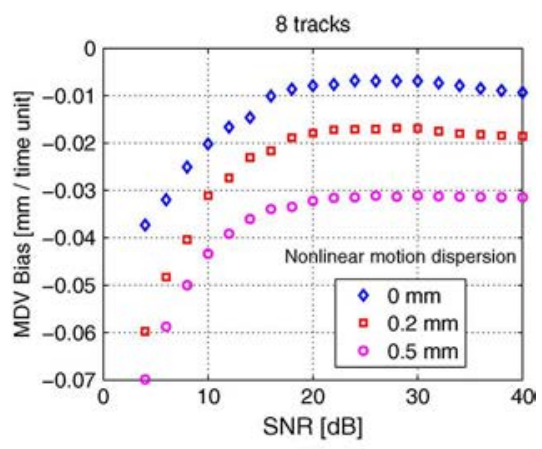

(d)

Fig. 11. Detection probability and MDV for one scatterer having a combination of linear and unmodeled nonlinear motion in LOS. The legend signifies the dispersion of the nonlinear motion. Detection probability for (a) 5 tracks and (b) 8 tracks. MDV (c) dispersion and (d) bias for 8 tracks.

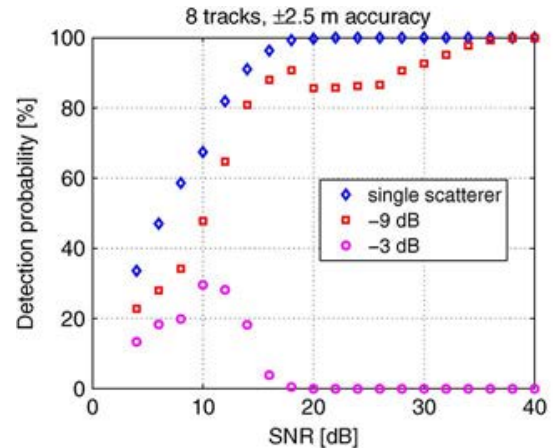

(a)

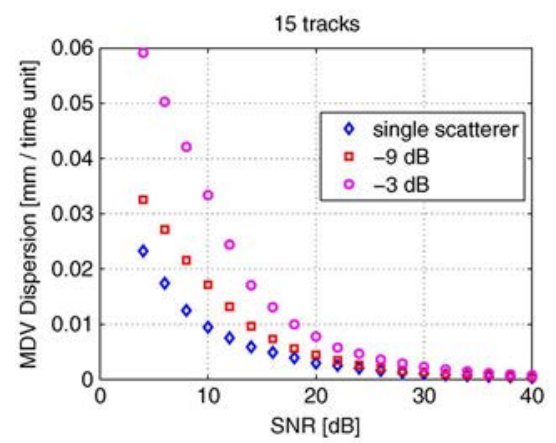

(c)

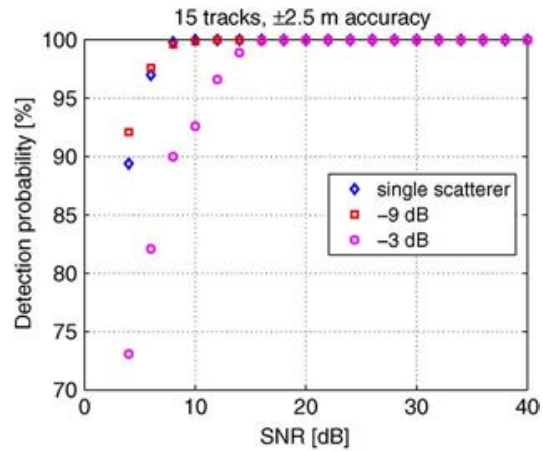

(b)

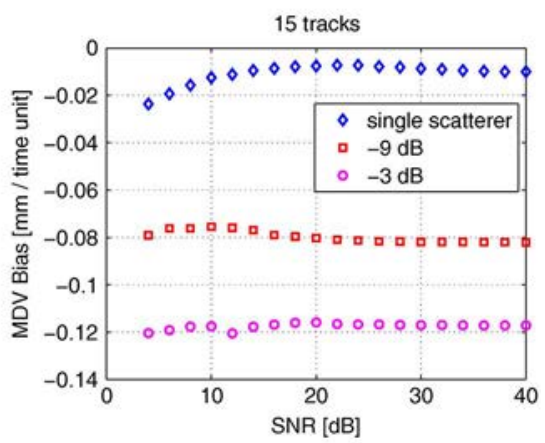

(d)

Fig. 12. Detection probability and MDV for two scatterers (a second target placed in layover with the main one). The legend signifies the amplitude of the second scatterer relative to the main one. Detection probability for (a) 8 tracks and (b) 15 tracks. MDV (c) dispersion and (d) bias for 15 tracks.

different bandwidths and PRFs even for the same area under similar conditions. Hence, the azimuth common-band filtering described in [1] is necessary before defocusing in order to have the same sliding azimuth bandwidth in all images. Additionally, between the actual bandpass filtering and the reramping (steps involved in the azimuth common-band filtering), an azimuth upsampling is applied to avoid the wrapping of the Doppler spectrum. 


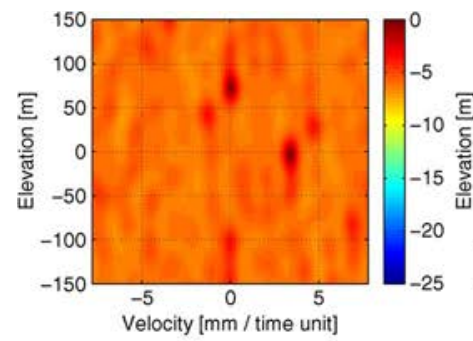

(a)

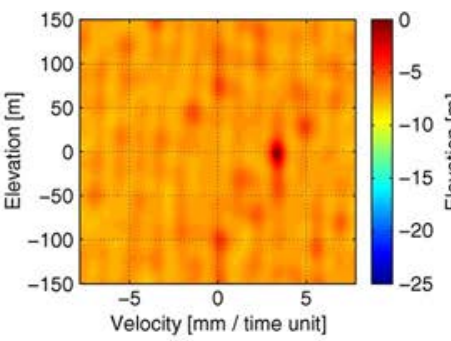

(b)

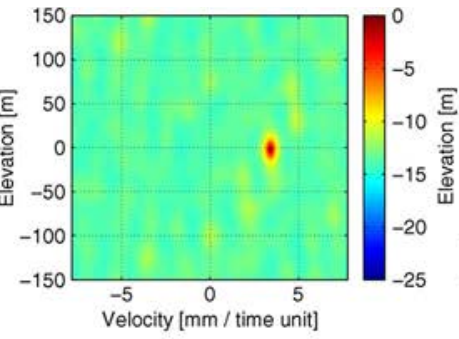

(c)

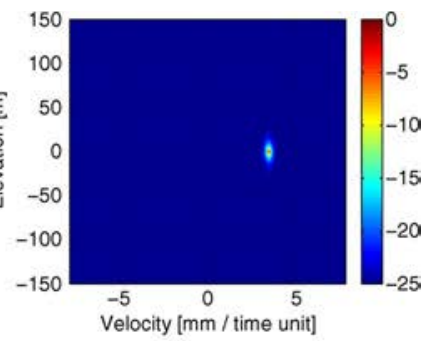

(d)

Fig. 13. Estimated EV PSD planes for 15 tracks, SNR of $20 \mathrm{~dB}$ and different amplitudes of the second scatterer relative to the main one: (a) $0 \mathrm{~dB}$, (b) $-3 \mathrm{~dB}$, (c) $-9 \mathrm{~dB}$, and (d) single scatterer.

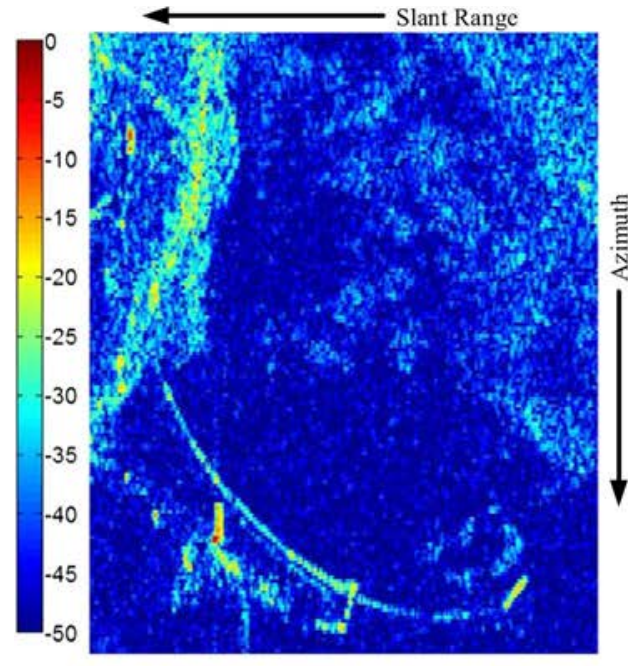

(a)

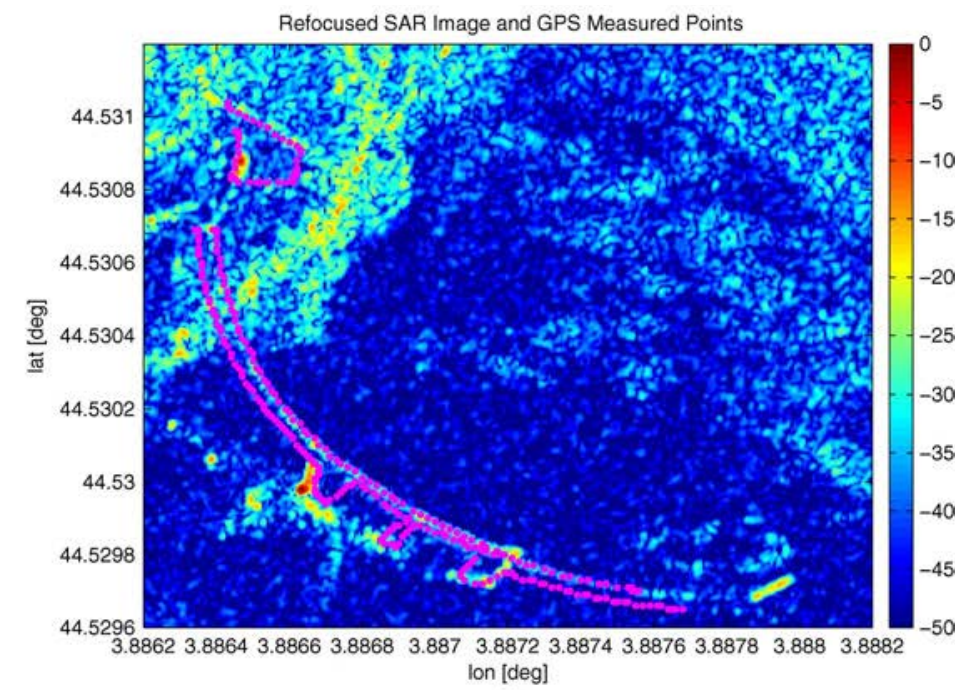

(b)

Fig. 14. Two-Dimensional Grid Refocusing. (a) High-Resolution Spotlight TerraSAR-X SAR image of the Puylaurent dam acquired on March 11, 2012. (b) Refocused TerraSAR-X image on the rectangular grid overlaid by the set of GPS measured points on the ridge of the dam.

In addition, in order to achieve an accuracy of the grid focusing in the centimeter level the distances computed in (16) and (17) have to be adjusted with the annotated range and azimuth corrections (atmospheric shift and azimuth timing offset) [22].

Three different geographical grids of the Puylaurent water dam were used for refocusing: a rectangular 2-D grid, an irregular 2-D grid and a high-resolution DEM. The rectangular 2-D grid is an uniformly spaced grid $(0.5 \mathrm{~m} \times 0.5 \mathrm{~m})$ aligned with the local latitude/longitude axis and situated at the height of the dam's ridge. The irregular 2-D grid consists of a set of differential GPS measured points on the water dam's ridge (the points were converted from latitude/longitude to ECEF coordinates considering that they are all at the ridge's altitude). The high-resolution DEM was obtained in a LIDAR measurement campaign conducted by the EDF company in July 2013 and is composed from a set of points having an average spacing of $0.5 \mathrm{~m}$ between them.

Fig. 14(a) shows an image acquired by the TerraSAR-X satellite on March 11, 2012. Fig. 15(a) displays the same image as Fig. 14(a), but transposed in order to be in visual accordance with the DEM image. The corresponding refocused image on the rectangular grid is shown in Fig. 14(b) overlaid by the set of
GPS measured points. The rectangular grid was used in order to check the consistency between the location of the water dam in the refocused image in latitude/longitude coordinates and the GPS measured position of the dam's ridge. In Fig. 15(b) is presented the refocused image on the LIDAR measured DEM. Notice that in the 3-D image the highly reflective regions from the initial image appear at different heights and only a part of them are actually provided by the dam's ridge.

In order to detect which points from the ridge are scattering centers, the 4-D tomographic detection and tracking procedure was applied on the points from the ridge area for the 3-D DEM.

For each refocused point, an additional phase difference was employed using as reference a stable point situated at the dam's edge in order to compensate any residual propagation effects. This approach works because the extent of the water dam's ridge is below $200 \mathrm{~m}$ and the atmospheric effects remain unchanged throughout the structure. In the case of larger infrastructures, the effects of the atmosphere would have to be estimated and separated.

The PSD for each point was computed using the Capon estimator. The covariance matrix was estimated on 9 neighboring points (an area of around $1.5 \mathrm{~m} \times 1.5 \mathrm{~m}$ ) in keeping with the number of looks used in the simulations. 


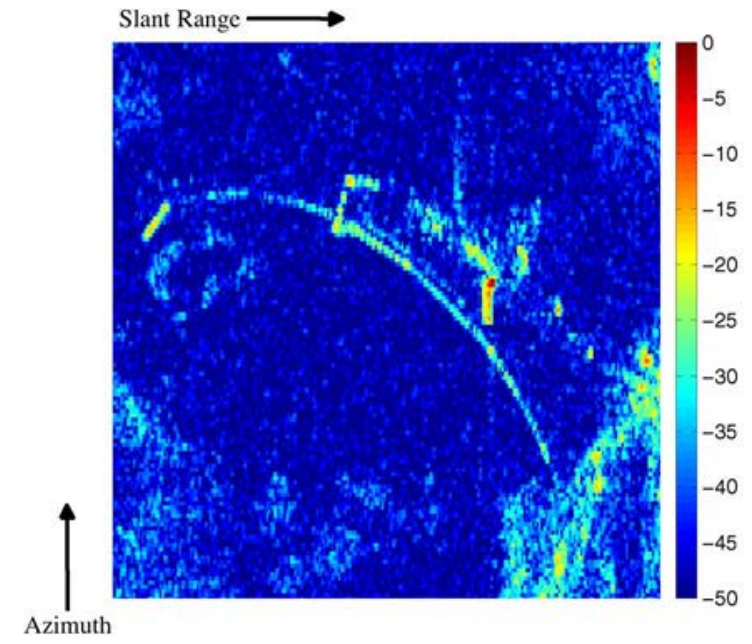

(a)

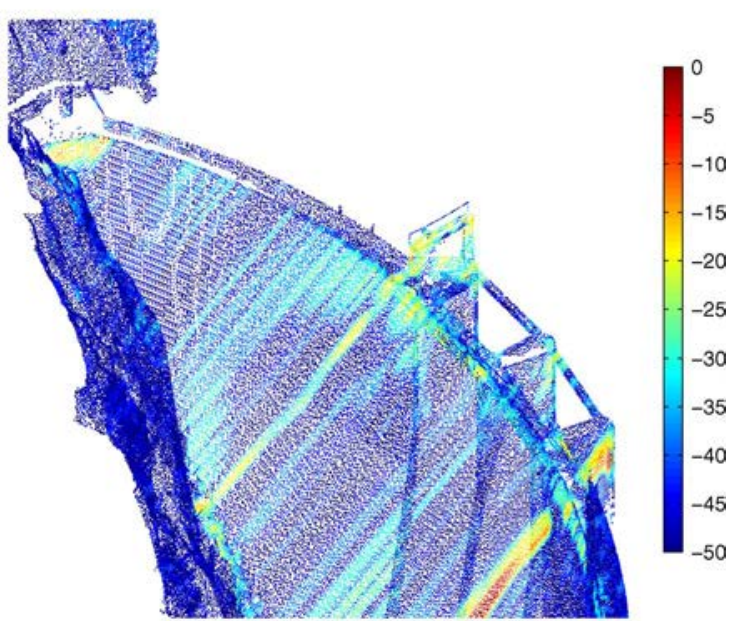

(b)

Fig. 15. Three-Dimensional Grid Refocusing. (a) The SAR image from Fig. 14(a) transposed to be visually consistent with the displayed DEM images. (b) Refocused TerraSAR-X image on the LIDAR measured DEM.

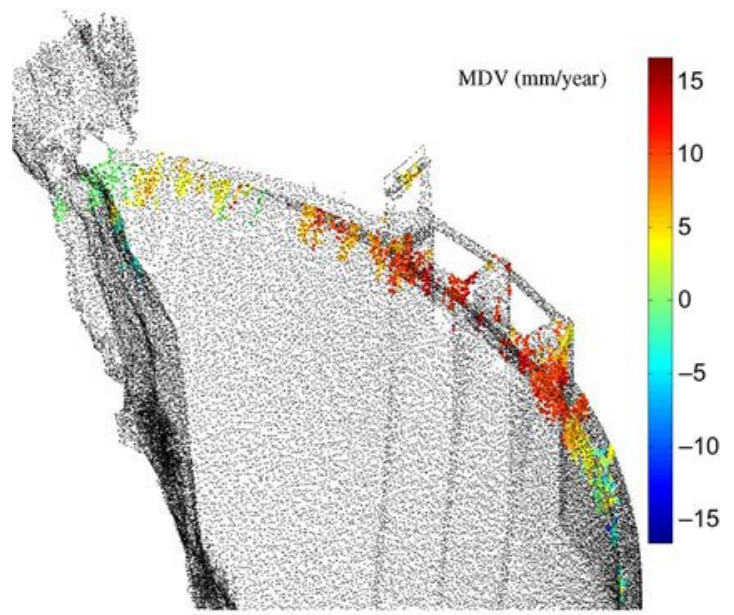

(a)

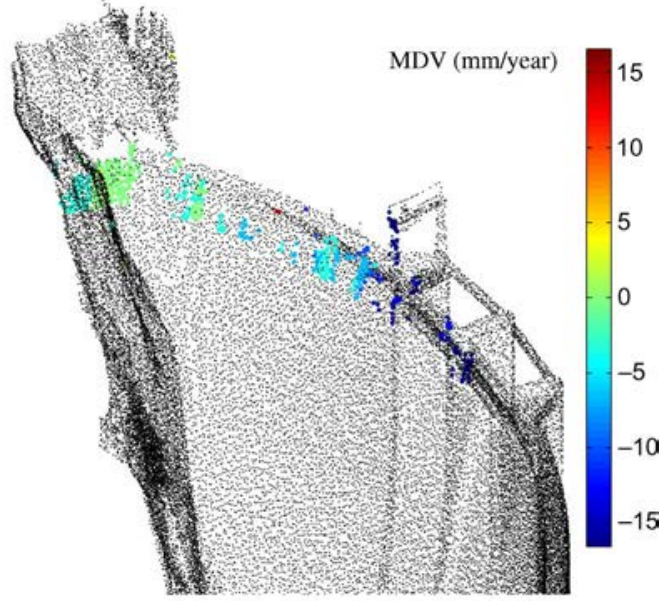

(b)

Fig. 16. Detected scattering centers and their MDV superimposed over the provided DEM of the Puylaurent dam for the two data sets: (a) May-June 2012, (b) April-October 2013. Positive/negative values mean expansion/contraction of the dam.

Fig. 16 shows for the two data sets the detected scattering centers of the DEM mapped according to their MDV. The positive and negative values indicate respectively expansion and contraction of the structure (this behavior is linked to the increase or respectively the decrease of the water level in the reservoir). The detected points are the ones which have the maximum PSD value around zero elevation with $\pm 2.5 \mathrm{~m}$ accuracy and SNR of at least $10 \mathrm{~dB}$ (the noise floor was considered the average level in the water near the dam). The points surrounding the dam's edge have the MDV equal to $0 \mathrm{~mm} / \mathrm{year}$ because this area was chosen as reference for the additional phase difference.

A typical normalized experimental PSD obtained in the EV plane for one given point in the presence of only one dominant scatterer is shown in Fig. 17. Notice that the main lobe is situated around zero elevation and is not dispersed on the velocity axis.

In order to validate the computed displacements, a comparison was made with in situ data. The water dam has five

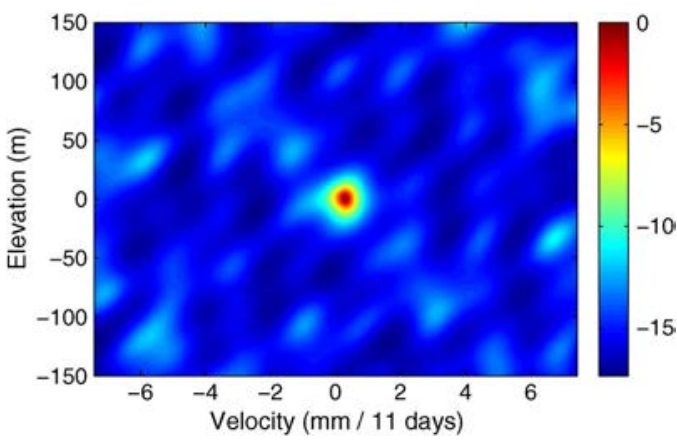

Fig. 17. Normalized experimental PSD in the EV plane for a refocused point having one dominant scatterer. The chosen time unit is the satellite's revisit time of 11 days.

pendulum systems which provide very accurate measurements of deformation $(0.01 \mathrm{~mm})$ between different regions of the dam's ridge. These measurements were projected in LOS and used as reference. However, not all the regions with pendulums 
TABLE II

Mean Displacement Velocity Measurements

\begin{tabular}{|c|c||c|c|}
\hline Data Set & Test Points & $\begin{array}{c}\text { Estimated } \\
(\mathrm{mm} / 11 \text { days })\end{array}$ & $\begin{array}{c}\text { In-situ } \\
(\mathrm{mm} / 11 \text { days })\end{array}$ \\
\hline 2012 & Central-left & 0.60 & 0.68 \\
\hline 2012 & Central-right & 0.35 & 0.33 \\
\hline 2013 & Central-right & -0.20 & -0.14 \\
\hline
\end{tabular}

are always detected as scattering centers; thus, the comparison with in situ data can be inherently limited. Table II shows a comparison between the estimated and in situ measured mean displacement velocities (using as time unit the satellite's revisit time of 11 days) in LOS for two detected scattering centers situated in different regions of the dam. According to the simulations from the previous section, an error below $0.1 \mathrm{~mm} /$ time unit is plausible for a SNR of around $15 \mathrm{~dB}$ and small data set (8 tracks).

The relative displacements time series were computed for the central-right region of the dam's ridge (looking from upstream to downstream) where the zero elevation peak was at least $10 \mathrm{~dB}$ higher than any other smaller peak of the EV plane. In this way only single scatterers are considered for displacements time series extraction. The obtained relative displacements between two locations on the dam's ridge in LOS for each satellite pass are presented in Fig. 18. The first pass was taken as reference and hence appears with zero displacement. The error is quite small $( \pm 0.5 \mathrm{~mm})$ and in keeping with the simulated dispersion.

\section{General Remarks}

Notice that the approach proposed in this paper differs from other works through several aspects both conceptual and in terms of implementation.

First of all, the usual approach for interferometric processing of SAR images is to identify the pixels which contain the response of stable natural reflectors or permanent scatters [30]-[32] and afterward to link its position to a possible known scatterer on the ground. This linking is done either by knowing a scatter's approximative position (e.g., a corner reflector mounted in a weakly reflecting environment) or by explicit geocoding. In our paper, we propose a reversed approach. Having a structure given in a certain way (a regular/irregular grid or a DEM) and refocusing the SAR image on it, we observe which points of the structure provide a response to the satellite flying on a specific orbit. Additionally, from the implementation point of view, by employing the refocusing approach for each acquired TerraSAR-X image, the coregistration of the SAR images [33], [34] is implicitly performed with an accuracy in the centimeter range due to the excellent ranging information provided by TerraSAR-X [8].

The original version of the back-projection algorithm [26] is used to focus raw data with possible range cell migration, whereas the one used in this paper is adapted to range compressed-azimuth defocused data, where the phase history of a target is on a single column of the data matrix.

In works concerning multiple scatterers monitoring in the same pixel [6], [7], [35]-[37] the 4-D tomography framework is used to compute the position of different scatterers in elevation
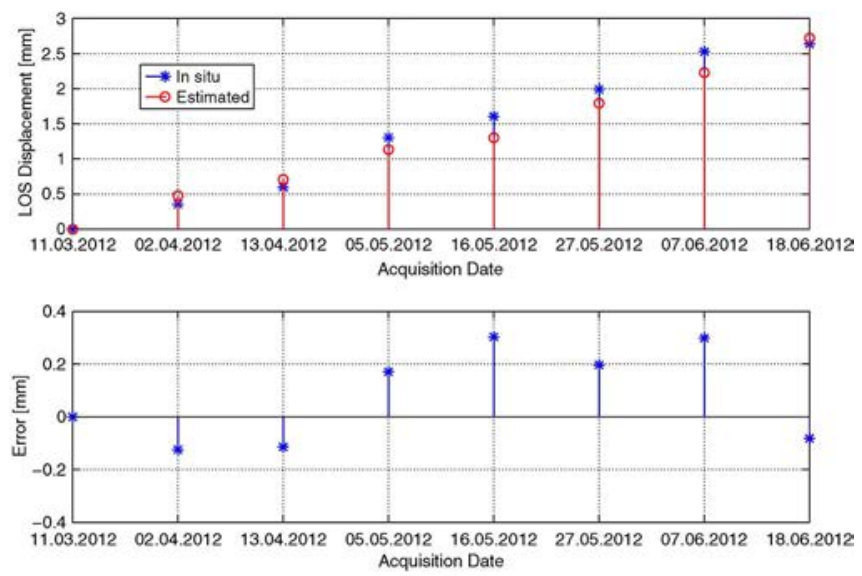

(a)
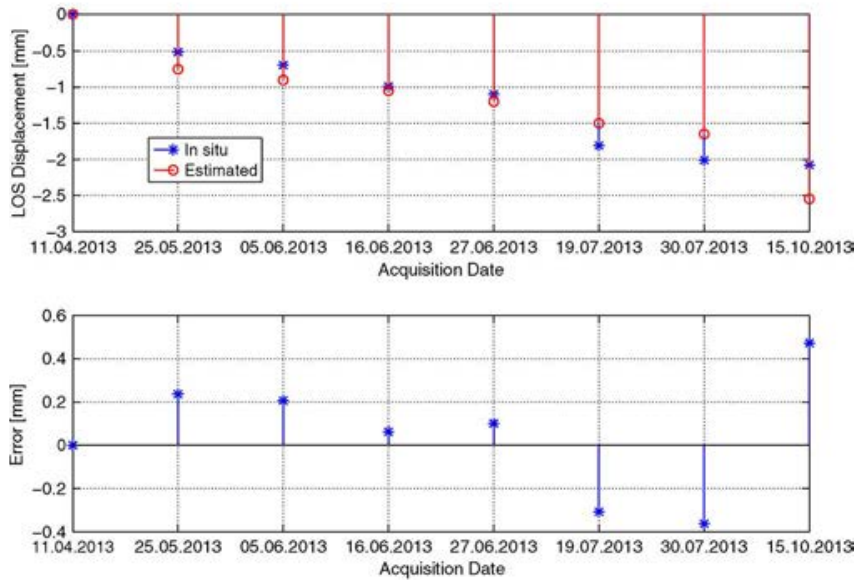

(b)

Fig. 18. Estimated versus in situ LOS displacements between two regions of the Puylaurent dam for the two data sets: (a) May-June 2012, (b) April-October 2013.

relative to a reference surface and the baselines projections are the same for an azimuth line (containing pixels with same slant range). On the other hand, in our approach the differential tomography is employed to test if a given point from a grid is indeed a scatterer. Moreover, the baselines projections change from point to point in order to maintain the zero elevation property of on-grid targets.

For more complex nonlinear movement which spreads the response of a target in the EV plane an on-grid scatterer may not be correctly detected by the proposed method. Still, if the movement follows a particular model, its parameters may be integrated in the framework and estimated in an Elevation-New Parameters space using an approach like the time warp proposed in [38]. However, this is beyond the scope of this paper.

\section{CONCLUSION}

A 4-D tomography-based scattering centers detection and tracking procedure for refocused SAR images on a provided grid has been presented. The performances and limitations of the method were analyzed by simulations for various scenarios. The method is well suited for detecting slowly moving scattering centers from a provided 3-D grid, selecting the scatterers unaffected by layover and computing their displacements. 
The efficiency of the method was tested on high-resolution sliding spotlight SAR images acquired with the TerraSAR-X satellite over the Puylaurent dam in France. The computed relative displacements for the detected scattering regions were in very good agreement with in situ data.

In perspective, future work will be centered on enhancing the refocusing method performances by applying specific timefrequency signal processing tools on the azimuth defocused signal.

As a long-term application, the developed technique will be used in civil engineering to monitor structures that do not have an in situ measuring system, but for which an accurate 3-D model is available.

\section{ACKNOWLEDGMENT}

The authors would like to thank the German Aerospace Center (DLR) for providing the TerraSAR-X and TanDEM-X SAR images through the MTH0828 and the NTIINSA2171 projects. The authors also thank Dr. I. Hajnsek (DLR) and Dr. P. Prats (DLR) for the useful discussions regarding the refocusing of high-resolution spotlight SAR images acquired in the TanDEM-X mission. The authors would also like to thank Rémy Boudon, Guy d'Urso and Didier Boldo from the Électricité de France (EDF) company for providing the in situ measurements for the Puylaurent dam. The authors also thank the anonymous reviewers for their helpful suggestions.

\section{REFERENCES}

[1] M. Eineder, N. Adam, R. Bamler, N. Yague-Martinez, and H. Breit, "Spaceborne spotlight SAR interferometry with TerraSAR-X," IEEE Trans. Geosci. Remote Sens., vol. 47, no. 5, pp. 1524-1535, May 2009.

[2] T. Fritz et al., "Interferometric processing of TanDEM-X data," in Proc. IEEE IGARSS, 2011, pp. 2428-2431.

[3] A. Anghel et al., "SAR images refocusing and scattering center detection for infrastructure monitoring," in Proc. IEEE Radar Conf., Cincinnati, OH, USA, May 2014, pp. 334-339.

[4] A. Anghel et al., "Scattering centers monitoring in refocused SAR images on a high-resolution DEM," in Proc. IEEE IGARSS, 2014, pp. 1883-1886.

[5] F. Lombardini, "Differential tomography: a new framework for SAR interferometry," IEEE Trans. Geosci. Remote Sens., vol. 43, no. 1, pp. 37-44, Jan. 2005

[6] G. Fornaro, D. Reale, and F. Serafino, "Four-dimensional SAR imaging for height estimation and monitoring of single and double scatterers," IEEE Trans. Geosci. Remote Sens., vol. 47, no. 1, pp. 224-237, Jan. 2009.

[7] F. Lombardini and M. Pardini, "Superresolution differential tomography: Experiments on identification of multiple scatterers in spaceborne SAR data," IEEE Trans. Geosci. Remote Sens., vol. 50, no. 4, pp. 1117-1129, Apr. 2012

[8] T. Fritz, H. Breit, and M. Eineder, "TerraSAR-X products-tips and tricks," presented at the TerraSAR-X Science Meet., Oberpfaffenhofen, Germany, 2008. [Online]. Available: http://terrasar-x.dlr.de/papers_sci_ meet_3/tricks/Tutorial_SAR_Breit.pdf

[9] S. Ugur, O. Arikan, and A. Gurbuz, "Off-grid sparse SAR image reconstruction by EMMP algorithm," in Proc. IEEE Radar Conf., 2013, pp. 1-4.

[10] A. De Maio, G. Fornaro, and A. Pauciullo, "Detection of single scatterers in multidimensional SAR imaging," IEEE Trans. Geosci. Remote Sens., vol. 47, no. 7, pp. 2284-2297, Jul. 2009.

[11] A. Pauciullo, D. Reale, A. De Maio, and G. Fornaro, "Detection of double scatterers in SAR tomography," IEEE Trans. Geosci. Remote Sens., vol. 50, no. 9, pp. 3567-3586, Sep. 2012.

[12] D. Reale, G. Fornaro, and A. Pauciullo, "Extension of 4-D SAR imaging to the monitoring of thermally dilating scatterers," IEEE Trans. Geosci. Remote Sens., vol. 51, no. 12, pp. 5296-5306, Dec. 2013.

[13] A. Moreira, J. Mittermayer, and R. Scheiber, "Extended chirp scaling algorithm for air- and spaceborne SAR data processing in stripmap and
ScanSAR imaging modes," IEEE Trans. Geosci. Remote Sens., vol. 34, no. 5, pp. 1123-1136, Sep. 1996.

[14] J. Mittermayer, A. Moreira, and O. Loffeld, "Spotlight SAR data processing using the frequency scaling algorithm," IEEE Trans. Geosci. Remote Sens., vol. 37, no. 5, pp. 2198-2214, Sep. 1999.

[15] A. Moreira, "Real-time Synthetic Aperture Radar (SAR) processing with a new subaperture approach," IEEE Trans. Geosci. Remote Sens., vol. 30, no. 4, pp. 714-722, Jul. 1992.

[16] J. Mittermayer, R. Lord, and E. Borner, "Sliding spotlight SAR processing for TerraSAR-X using a new formulation of the extended chirp scaling algorithm," in Proc. IEEE IGARSS, Jul. 2003, vol. 3, pp. 1462-1464.

[17] P. Prats, R. Scheiber, J. Mittermayer, A. Meta, and A. Moreira, "Processing of sliding spotlight and TOPS SAR data using baseband azimuth scaling," IEEE Trans. Geosci. Remote Sens., vol. 48, no. 2, pp. 770-780, Feb. 2010.

[18] M. Sack, M. Ito, and I. Cumming, "Application of efficient linear FM matched filtering algorithms to synthetic aperture radar processing," Proc. Inst. Elect. Eng.-F Commun., Radar Signal Process., vol. 132, no. 1, pp. 45-57, Feb. 1985

[19] W. G. Carrara, R. S. Goodman, and R. M. Majewski, Spotlight Synthetic Aperture Radar: Signal Processing Algorithms. Boston, MA, USA: Artech House, 1995.

[20] M. Born and E. Wolf, Principles of Optics. Cambridge, U.K.: Cambridge Univ. Press, 1999, ch. Appendix 3.

[21] R. Raney, "A new and fundamental Fourier transform pair," in Proc. IGARSS, 1992, vol. 1, pp. 106-107.

[22] T. Fritz, M. J., B. Schättler, B. W., S. Buckreuß, and R. Werninghaus, "TerraSAR-X ground segment level $1 \mathrm{~b}$ product format specification," DLR, Cologne, German, 2007. [Online]. Available: http://terrasarx.dlr.de/

[23] P. Prats-Iraola et al., "High precision SAR focusing of TerraSAR$\mathrm{X}$ experimental staring spotlight data," in Proc. IEEE IGARSS, 2012, pp. 3576-3579.

[24] D. C. Munson, Jr., J. O’Brien, and W. Jenkins, "A tomographic formulation of spotlight-mode synthetic aperture radar," Proc. IEEE, vol. 71, no. 8, pp. 917-925, Aug. 1983.

[25] M. Desai and W. Jenkins, "Convolution backprojection image reconstruction for spotlight mode synthetic aperture radar," IEEE Trans. Image Process., vol. 1, no. 4, pp. 505-517, Oct. 1992.

[26] L. A. Gorham and L. J. Moore, "SAR image formation toolbox for MATLAB," Proc. SPIE, vol. 7699, 2010, Art. ID. 769906.

[27] M. Albuquerque, P. Prats, and R. Scheiber, "Applications of time-domain back-projection SAR processing in the airborne case," in Proc. 7th EUSAR, June 2008, pp. 1-4.

[28] J. Capon, "High-resolution frequency-wavenumber spectrum analysis," Proc. IEEE, vol. 57, no. 8, pp. 1408-1418, Aug.1969.

[29] H. Van Trees, Detection, Estimation, and Modulation Theory: Optimum Array Processing. New York, NY, USA: Wiley-Interscience, 2002.

[30] A. Ferretti, C. Prati, and F. Rocca, "Permanent scatterers in SAR interferometry," IEEE Trans. Geosci. Remote Sens., vol. 39, no. 1, pp. 8-20, Jan. 2001.

[31] C. Colesanti, A. Ferretti, F. Novali, C. Prati, and F. Rocca, "SAR monitoring of progressive and seasonal ground deformation using the permanent scatterers technique," IEEE Trans. Geosci. Remote Sens., vol. 41, no. 7, pp. 1685-1701, Jul. 2003.

[32] A. Anghel, G. Vasile, J.-P. Ovarlez, G. D'Urso, and D. Boldo, "Stable scatterers detection and tracking in heterogeneous clutter by repeat-pass SAR interferometry," in Proc. 9th Eur. Conf. Synth. Aperture Radar, Apr. 2012, pp. 477-480.

[33] F. Serafino, "SAR image coregistration based on isolated point scatterers," IEEE Geosci. Remote Sens. Lett., vol. 3, no. 3, pp. 354-358, Jul. 2006.

[34] R. Scheiber and A. Moreira, "Coregistration of interferometric SAR images using spectral diversity," IEEE Trans. Geosci. Remote Sens., vol. 38, no. 5, pp. 2179-2191, Sep. 2000

[35] S. Sauer, L. Ferro-Famil, A. Reigber, and E. Pottier, "Three-dimensional imaging and scattering mechanism estimation over urban scenes using dual-baseline polarimetric InSAR observations at L-Band," IEEE Trans. Geosci. Remote Sens., vol. 49, no. 11, pp. 4616-4629, Nov. 2011.

[36] M. Nannini, R. Scheiber, and A. Moreira, "Estimation of the minimum number of tracks for SAR tomography," IEEE Trans. Geosci. Remote Sens., vol. 47, no. 2, pp. 531-543, Feb. 2009.

[37] X. X. Zhu and R. Bamler, "Tomographic SAR inversion by $L_{1}$-Norm regularization-the compressive sensing approach," IEEE Trans. Geosci. Remote Sens., vol. 48, no. 10, pp. 3839-3846, Oct. 2010.

[38] X. X. Zhu and R. Bamler, "Let's do the time warp: Multicomponent nonlinear motion estimation in differential SAR tomography," IEEE Geosci. Remote Sens. Lett., vol. 8, no. 4, pp. 735-739, Jul. 2011 